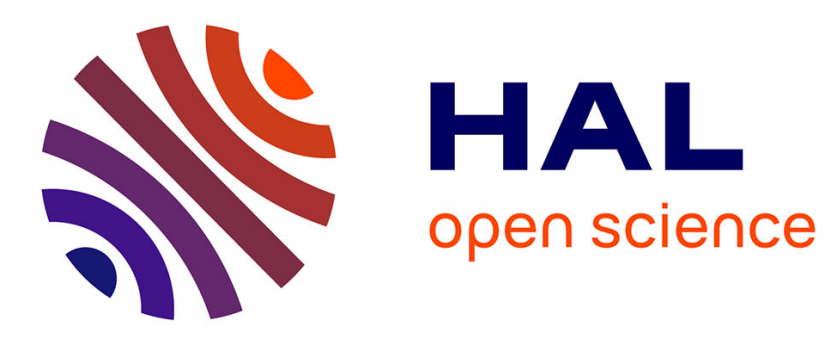

\title{
Good edge-labelling of graphs
}

Julio Araújo, Nathann Cohen, Frédéric Giroire, Frédéric Havet

\section{To cite this version:}

Julio Araújo, Nathann Cohen, Frédéric Giroire, Frédéric Havet. Good edge-labelling of graphs. [Research Report] RR-6934, INRIA. 2009, pp.16. inria-00383343v4

\section{HAL Id: inria-00383343 https://hal.inria.fr/inria-00383343v4}

Submitted on 15 May 2009

HAL is a multi-disciplinary open access archive for the deposit and dissemination of scientific research documents, whether they are published or not. The documents may come from teaching and research institutions in France or abroad, or from public or private research centers.
L'archive ouverte pluridisciplinaire HAL, est destinée au dépôt et à la diffusion de documents scientifiques de niveau recherche, publiés ou non, émanant des établissements d'enseignement et de recherche français ou étrangers, des laboratoires publics ou privés. 
INSTITUT NATIONAL DE RECHERCHE EN INFORMATIQUE ET EN AUTOMATIQUE

\title{
Good edge-labelling of graphs
}

\author{
J. Araujo — N. Cohen - F. Giroire — F. Havet
}

\section{$\mathbf{N}^{\circ} 6934$}

May 15, 2009

Thème COM

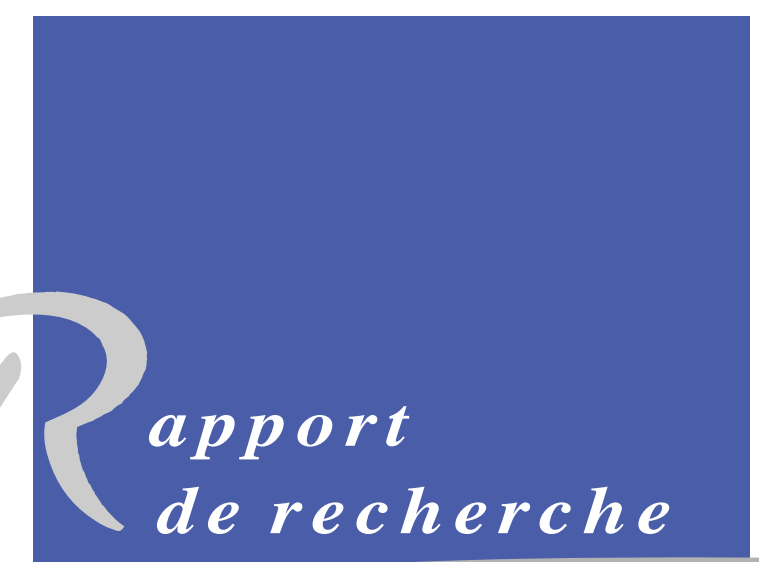





\title{
RIN RIA
}

\section{Good edge-labelling of graphs}

\author{
J. Araujo , N. Cohen , F. Giroire , F. Havet \\ Thème COM — Systèmes communicants \\ Projets Mascotte
}

Rapport de recherche $n^{\circ} 6934$ - May 15, 2009 - 19 pages

\begin{abstract}
A good edge-labelling of a graph $G$ is a labelling of its edges such that, for any ordered pair of vertices $(x, y)$, there do not exist two paths from $x$ to $y$ with increasing labels. This notion was introduced in [?] to solve wavelength assignment problems for specific categories of graphs. In this paper, we aim at characterizing the class of graphs that admit a good edge-labelling. First, we exhibit infinite families of graphs for which no such edgelabelling can be found. We then show that deciding if a graph admits a good edge-labelling is NP-complete. Finally, we give large classes of graphs admitting a good edge-labelling: forests, $C_{3}$-free outerplanar graphs, planar graphs of girth at least 6 , subcubic $\left\{C_{3}, K_{2,3}\right\}$-free graphs.
\end{abstract}

Key-words: graph theory, complexity, edge-labelling, planar graphs, matching-cut, channel assignment. 


\section{Bon étiquetages des arêtes des graphes}

Résumé : Un bon étiquetage des arêtes d'un graphe est un étiquetage de ses arêtes tel que, pour toute paire de sommets $(x, y)$, il n'existe pas deux chemins de $x$ vers $y$ dont les étiquettes sont croissantes. Cette notion a été introduite dans [?] pour résoudre des problèmes d'allocation de fréquence pour des classes particulières de graphes. Dans cet article, nous tentons de caractériser la classe des graphes qui admettent un bon étiquetage. Tout d'abord, nous produisons des familles infinies de graphes pour lesquelles aucun bon étiquetage n'existe. Nous montrons par la suite que le problème de décider si un graphe admet un bon étiquetage est NP-Complet. Finalement, nous donnons de larges classes de graphes admettant un bon étiquetage : les forêts, les graphes sans triangles, les graphes planaires de maille au moins 6 , les graphes subcubiques sans triangles ni $K_{2,3}$

Mots-clés : théorie des graphes, complexité, coloration d'arêtes, graphes planaires, coupe parallle, allocation de fréquence. 


\title{
Good edge-labelling of graphs
}

\author{
J. Araujo, N. Cohen, F. Giroire and F. Havet
}

May 15, 2009

A good edge-labelling of a graph $G$ is a labelling of its edges such that, for any ordered pair of vertices $(x, y)$, there do not exist two paths from $x$ to $y$ with increasing labels. This notion was introduced in [2] to solve wavelength assignment problems for specific categories of graphs. In this paper, we aim at characterizing the class of graphs that admit a good edge-labelling. First, we exhibit infinite families of graphs for which no such edge-labelling can be found. We then show that deciding if a graph admits a good edge-labelling is NPcomplete. Finally, we give large classes of graphs admitting a good edge-labelling: forests, $C_{3}$-free outerplanar graphs, planar graphs of girth at least 6 , subcubic graphs.

\section{Introduction}

A classical and widely studied problem in WDM (Wavelength Division Multiplexing) networks is the Routing and Wavelength Assignment (RWA) problem [8, 9, 1]. It consists in finding routes, and their associated wavelength as well, to satisfy a set of traffic requests while minimising the number of used wavelengths. This is a difficult problem which is, in general, NP-hard. Thus, it is often split into two distinct problems: First, routes are found for the requests. Then, in a second step, these routes are taken as an input. Wavelengths must be associated to them in such a way that two routes using the same fiber do not have the same wavelength. The last problem can be reformulated as follows: Given a digraph and a set of dipaths, corresponding to the routes for the requests, find the minimal number of wavelengths $w$ needed to assign different wavelengths to dipaths sharing an edge. This problem can be seen as a colouring problem of the conflict graph which is defined as follows: It has one vertex per dipath and two vertices are linked by an edge if their corresponding dipaths share an edge. In [2], Bermond et al. studied the RWA problem for UPP-DAG which are acyclic digraphs (or DAG) in which there is at most one dipath from one vertex to another. In such digraph the routing is forced and thus the unique problem is the wavelength assignment one.

In their paper, they introduce the notion of good edge-labelling. An edge-labelling of a graph $G$ is a function $\phi: E(G) \rightarrow \mathbb{R}$. A path is increasing if the sequence of its edge labels is non-decreasing. An edge-labelling of $G$ is good if, for any two distinct vertices $u, v$, there is at most one increasing $(u, v)$-path. Bermond et al. [2] showed that the conflict graph of a 
set of dipaths in a UPP-DAG has a good edge-labelling. Conversely, for any graph admitting a good edge-labelling one can exhibit a family of dipaths on a UPP-DAG whose conflict graph is precisely this graph. Bermond et al. [2] then use the existence of graphs with a good edge-labelling and large chromatic number to prove that there exist sets of requests on UPP-DAGs with load 2 (an edge is shared by at most two paths) requiring an arbitrarily large number of wavelengths.

To obtain other results on this problem, it may be useful to identify the good graphs which admit a good labelling and the bad ones which do not. Bermond et al.[2] noticed that $C_{3}$ and $K_{2,3}$ are bad. J.-S. Sereni [11] asked whether every $\left\{C_{3}, K_{2,3}\right\}$-free graph (i.e., with no $C_{3}$ nor $K_{2,3}$ as a subgraph) is good. In Section 3 , we answer this question in the negative. We give an infinite family of bad graphs none of which is the subgraph of another.

Furthermore, in Section 4, we prove that determining if a graph has a good edge-labelling is NP-complete using a reduction of Not-All-Equal 3-SAT.

In Section 5, we show large classes of good graphs: forests, $C_{3}$-free outerplanar graphs, planar graphs of girth at least 6 . To do so, we use the notion of critical graph which is a bad graph every proper subgraph of which is good. Clearly, a good edge-labelling of a graph induces a good edge-labelling of all its subgraphs. So every bad graph must contain a critical subgraph. We establish several properties of critical graphs. In particular, we show that they have no matching-cut. Hence, a result of Farley and Proskurowski [6] (Theorem 16) implies that a critical graph $G$ has a least $\frac{3}{2}|V(G)|-\frac{3}{2}$ edges unless $G$ is $C_{3}$ or $K_{2,3}$.

In Section 6, we use the characterization of graphs with no matching-cut and $\left\lceil\frac{3}{2}|V(G)|-\frac{3}{2}\right\rceil$ edges given by Bonsma [3] to slightly improve this result. We show that a critical graph $G$ has a least $\frac{3}{2}|V(G)|-\frac{1}{2}$ edges unless $G$ is $C_{3}$ or $K_{2,3}$.

Finally, we present avenues for future research.

\section{Preliminaries}

In this section, we give some technically useful propositions. Their proof are straightforward and left to the reader.

A path is decreasing if the sequence of its edge labels is non-increasing. Then, a path $u_{1} u_{2} \ldots u_{k}$ is decreasing if and only if its reversal $u_{k} u_{k-1} \ldots u_{1}$ is increasing. Hence an edge-labelling is good if and only if for any two distinct vertices $u, v$, there is at most one decreasing $(u, v)$-path. Equivalently, an edge-labelling is good if and only if for any two distinct vertices $u, v$, there is at most one increasing $(u, v)$-path and at most one decreasing $(u, v)$-path.

Let $x$ and $y$ be two vertices of $G$. Two distinct $(x, y)$-paths $P$ and $Q$ are independent if $V(P) \cap V(Q)=\{x, y\}$. Observe that in an edge-labelled graph $G$, there are two vertices $(u, v)$ with two increasing $(u, v)$-paths if and only if there are two vertices $(u, v)$ with two increasing independent $(u, v)$-paths. Hence the definition of good edge-labelling may be expressed in terms of independent paths.

Proposition 1 An edge-labelling is good if and only if for any two distinct vertices $u$ and $v$, there are no two increasing independent $(u, v)$-paths. 
As above the definition may also be in terms of decreasing independent paths.

Proposition 2 Let $G$ be a graph. Then the following assertions are equivalent:

(i) G admits a good edge-labelling in $\mathbb{R}$;

(ii) $G$ admits an injective good edge-labelling;

(iii) $G$ admits a good edge-labelling in $\mathbb{R} \cup\{-\infty,+\infty\}$;

(iv) $G$ admits a good edge-labelling in $\mathbb{Z}$.

In the paper, we sometimes use these propositions without referring explicitly to them.

\section{Bad graphs}

A path of length one is both increasing and decreasing, and a path of length two is either increasing or decreasing. So $C_{3}$ has clearly no good edge-labelling. Also $K_{2,3}$ does not admit a good edge-labelling since there are three paths of length two between the two vertices of degree 2. Hence, in any edge-labelling, two of them are increasing or two of them are decreasing.

Extending this idea, we now construct an infinite family of bad graphs, none of which is the subgraph of another. The construction of this family is based on the graphs $H_{k}$ defined below. These graphs play the same role as a path of length two because they have two vertices $u$ and $v$ such that any good edge-labelling of $H_{k}$ has either a $(u, v)$-increasing path or a $(v, u)$-increasing path.

For any integer $k \geq 3$, let $H_{k}$ be the graph defined by

$$
\begin{aligned}
V\left(H_{k}\right)= & \{u, v\} \cup\left\{u_{i} \mid 1 \leq i \leq k\right\} \cup\left\{v_{i} \mid 1 \leq i \leq k\right\}, \\
E\left(H_{k}\right)= & \left\{u u_{i} \mid 1 \leq i \leq k\right\} \cup\left\{u_{i} v_{i} \mid 1 \leq i \leq k\right\} \cup\left\{v_{i} v \mid 1 \leq i \leq k\right\}, \\
& \cup\left\{v_{i} u_{i+1} \mid 1 \leq i \leq k\right\}
\end{aligned}
$$

with $u_{k+1}=u_{1}$. See Figure 1 .

Observe that the graph $H_{k}$ has no $K_{2,3}$ as a subgraph.

Proposition 3 Let $k \geq 3$. For every good edge-labelling, the graph $H_{k}$ has either an increasing $(u, v)$-path or an increasing $(v, u)$-path.

Proof: Suppose, by way of contradiction, that $H_{k}$ has a good edge-labelling $\phi$ having no increasing $(u, v)$-path and no increasing $(v, u)$-path. By Proposition 2, we may assume that $\phi$ is injective.

A key component in this proof is the following observation which follows easily from the fact that $\phi$ is good.

Observation 3.1 If $x_{1} x_{2} x_{3} x_{4} x_{1}$ is a 4-cycle. Then, either $\phi\left(x_{4} x_{1}\right)<\phi\left(x_{1} x_{2}\right), \phi\left(x_{2} x_{3}\right)<$ $\phi\left(x_{1} x_{2}\right), \phi\left(x_{2} x_{3}\right)<\phi\left(x_{3} x_{4}\right), \phi\left(x_{1} x_{4}\right)<\phi\left(x_{3} x_{4}\right)$, or all those inequalities are reversed. 


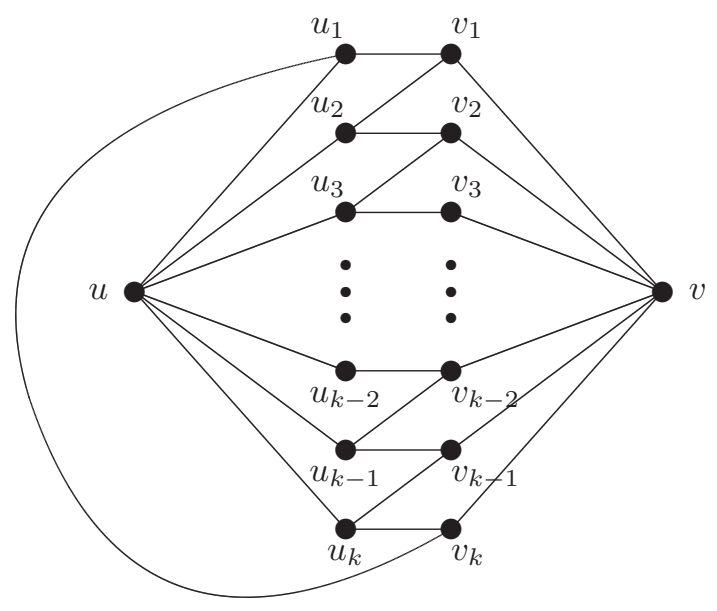

Figure 1: Graph $H_{k}$

By symmetry, we may assume that $\phi\left(u u_{1}\right)<\phi\left(u_{1} v_{1}\right)$. By Observation 3.1, $\phi\left(v_{1} u_{2}\right)<$ $\phi\left(u u_{2}\right)$ and $\phi\left(u u_{1}\right)<\phi\left(u u_{2}\right)$. Then, since $v v_{1} u_{2} v_{1} u$ is not increasing, $\phi\left(u_{2} v_{1}\right)<\phi\left(v_{1} v\right)$. Again by Observation 3.1, $\phi\left(v_{2} v\right)<\phi\left(u_{2} v_{2}\right)$. Thus since $u u_{2} v_{2} v$ is not increasing $\phi\left(u u_{2}\right)<$ $\phi\left(u_{2} v_{2}\right)$.

Applying the same reasoning iteratively, we obtain that $\phi\left(u u_{2}\right)<\phi\left(u u_{3}\right)$ and $\phi\left(u u_{3}\right)<$ $\phi\left(u_{3} v_{3}\right)$ And so on, iteratively, $\phi\left(u u_{1}\right)<\phi\left(u u_{2}\right)<\cdots<\phi\left(u u_{k}\right)<\phi\left(u u_{1}\right)$, a contradiction.

For convenience we denote by $H_{2}$ the path of length 2 with end vertices $u$ and $v$. Let $i, j, k$ be three integers greater than 1 . The graph $J_{i, j, k}$ is the graph obtained from disjoint copies of $H_{i}, H_{j}$ and $H_{k}$ by identifying the vertices $u$ of the three copies and the vertices $v$ of the three copies.

Proposition 4 Let $i, j, k$ be three integers greater than 1 . Then $J_{i, j, k}$ is bad.

Proof: Suppose, by way of contradiction, that $J_{i, j, k}$ admits a good edge-labelling. By Proposition 3, in each of the subgraphs $H_{i}, H_{j}$ and $H_{k}$, there is either an increasing $(u, v)$ path or an increasing $(v, u)$-path. Hence in $J_{i, j, k}$, there are either two increasing $(u, v)$-paths or two increasing $(v, u)$-paths, a contradiction. 


\section{$4 \quad N P$-completeness}

In this section, we prove that it is an $N P$-complete problem to decide if a graph admits a good edge-labelling. We give a reduction from the NOT-ALL-EQUAL (NAE) 3-SAT Problem [10] which is defined as follows:

Instance: A set $V$ of variables and a collection $\mathcal{C}$ of clauses over $V$ such that each clause has exactly 3 literals.

Question: Is there a truth assignment such that each clause has at least one true and at least one false literal?

Theorem 5 The following problem is NP-complete.

Instance: A graph $G$.

Question: Does $G$ have a good edge-labelling?

Proof: We will actually reduce to the NAE 3-SAT Problem without repetition (i.e. a variable appears at most once in each clause) which is equivalent to NAE 3-SAT Problem (with repetition). (For each variable $x$, we introduce two other variables $y$ and $z$. Then the second (third) occurrence of $x$ in a clause is replaced by $y(z)$. Then, $x, y, z$ are forced to have the same truth assignment by adding $\bar{x} \vee y \vee z, x \vee \bar{y} \vee z$, and $x \vee y \vee \bar{z}$ to the instance.)

Let $V=\left\{x_{1}, \ldots, x_{n}\right\}$ and $\mathcal{C}=\left\{C_{1}, \ldots, C_{m}\right\}$ be an instance $I$ of the NAE 3-SAT Problem without repetition. We shall construct a graph $G_{I}$ in such a way that $I$ has an answer yes for the NAE 3-SAT Problem if and only if $G$ has a good edge-labelling.

For each variable $x_{i}, 1 \leq i \leq n$, we create a variable graph $V G_{i}$ defined as follows (See Figure 2.):

$$
\begin{aligned}
V\left(V G_{i}\right)= & \left\{v_{k}^{i, j} \mid 1 \leq j \leq m, 1 \leq k \leq 4\right\} \cup\left\{r_{k}^{i, j} \mid 1 \leq j \leq m, 1 \leq k \leq 4\right\} \\
& \cup\left\{s_{k}^{i, j} \mid 1 \leq j \leq m, 1 \leq k \leq 4\right\} . \\
E\left(V G_{i}\right)= & \left\{v_{k}^{i, j} v_{k+1}^{i, j} \mid 1 \leq j \leq m, 1 \leq k \leq 3\right\} \cup\left\{v_{4}^{i, j} v_{1}^{i, j+1} \mid 1 \leq j \leq m-1\right\} \\
& \cup\left\{v_{k}^{i, j} r_{k}^{i, j} \mid 1 \leq j \leq m, 1 \leq k \leq 4\right\} \cup\left\{v_{k}^{i, j} s_{k}^{i, j} \mid 1 \leq j \leq m, 1 \leq k \leq 4\right\} \\
& \cup\left\{v_{4}^{i, j} r_{1}^{i, j} \mid 1 \leq j \leq m\right\} \cup\left\{v_{k}^{i, j+1} r_{k+1}^{i, j} \mid 1 \leq j \leq m-1,1 \leq k \leq 3\right\} \\
& \cup\left\{v_{4}^{i, j} s_{1}^{i, j} \mid 1 \leq j \leq m\right\} \cup\left\{v_{k}^{i, j+1} s_{k+1}^{i, j} \mid 1 \leq j \leq m-1,1 \leq k \leq 3\right\} .
\end{aligned}
$$

For each clause $C_{j}=l_{1} \vee l_{2} \vee l_{3}, 1 \leq j \leq m$, we create a clause graph $C G_{j}$ defined as follows (See Figure 3.):

$$
\begin{aligned}
& V\left(C G_{j}\right)=\left\{c^{j}, b_{1}^{j}, b_{2}^{j}, b_{3}^{j}\right\} \\
& E\left(C G_{j}\right)=\left\{c^{j} b_{1}^{j}, c^{j} b_{2}^{j}, c^{j} b_{3}^{j}\right\}
\end{aligned}
$$

Now, for each literal $l_{k}, 1 \leq k \leq 3$, if $l_{k}$ is the non-negated variable $x_{i}$, we identify $b_{k}^{j}, c^{j}$ and $b_{k+1}^{j}$ (index $k$ is taken modulo 3) with $v_{1}^{i, j}, v_{2}^{i, j}$ and $v_{3}^{i, j}$, respectively. Otherwise, if $l_{k}$ is the negated variable $\bar{x}_{i}$, we identify $b_{k}^{j}, c^{j}$ and $b_{k+1}^{j}$ with $v_{3}^{i, j}, v_{2}^{i, j}$ and $v_{1}^{i, j}$, respectively. 


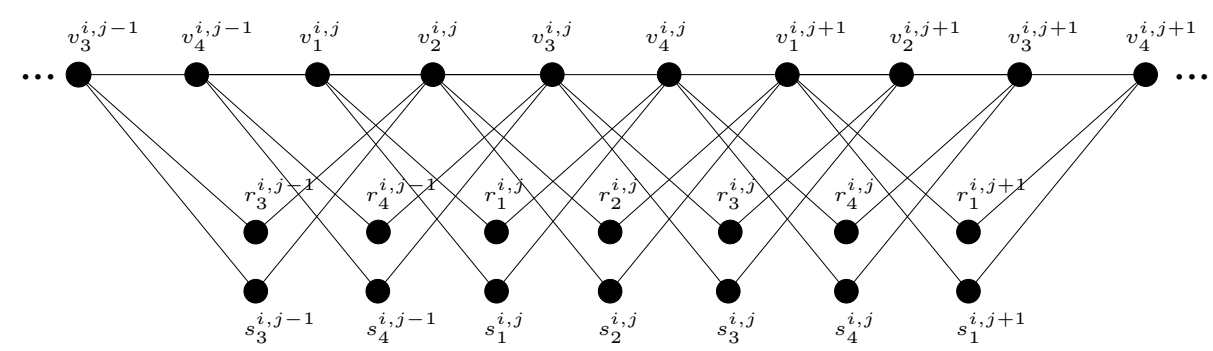

Figure 2: The variable graph $V G_{i}$

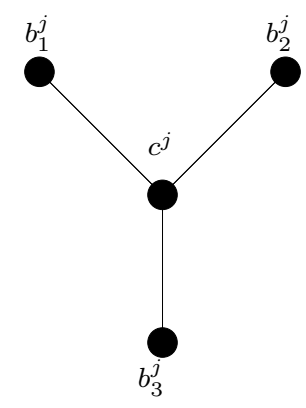

Figure 3: The clause graph $C G_{j}$. 
Let us first show that, if $G_{I}$ has a good edge-labelling $\phi$, then there is a truth assignment such that each clause of $I$ has at least one true literal and at least one false literal.

By Proposition 2, we may assume that $\phi$ is injective.

Claim 5.1 Let $1 \leq i \leq n$. If $\phi\left(v_{1}^{i, 1} v_{2}^{i, 1}\right)<\phi\left(v_{2}^{i, 1} v_{3}^{i, 1}\right)$ then $\phi\left(v_{1}^{i, j} v_{2}^{i, j}\right)<\phi\left(v_{2}^{i, j} v_{3}^{i, j}\right)$ for all $1 \leq j \leq m$.

Proof: By induction on $j$. A path of length two is necessarily increasing or decreasing. Now $v_{1}^{i, j}$ is joined to $v_{4}^{i, j}$ by two paths of length two via $r_{1}^{i, j}$ and $s_{1}^{i, j}$. Since $\phi$ is good, one of these two paths is increasing and the other one is decreasing. In addition, the path $v_{1}^{i, j} v_{2}^{i, j} v_{3}^{i, j} v_{4}^{i, j}$ is neither increasing nor decreasing so $\phi\left(v_{2}^{i, j} v_{3}^{i, j}\right)>\phi\left(v_{3}^{i, j} v_{4}^{i, j}\right)$.

Applying three times this reasoning, we derive $\phi\left(v_{3}^{i, j} v_{4}^{i, j}\right)<\phi\left(v_{4}^{i, j} v_{1}^{i, j+1}\right), \phi\left(v_{4}^{i, j} v_{1}^{i, j+1}\right)>$ $\phi\left(v_{1}^{i, j+1} v_{2}^{i, j+1}\right)$ and finally $\phi\left(v_{1}^{i, j+1} v_{2}^{i, j+1}\right)<\phi\left(v_{2}^{i, j+1} v_{3}^{i, j+1}\right)$.

Hence we define the truth assignment $\Lambda$ by $\Lambda\left(x_{i}\right)=\operatorname{true}$ if $\phi\left(v_{1}^{i, 1} v_{2}^{i, 1}\right)<\phi\left(v_{2}^{i, 1} v_{3}^{i, 1}\right)$ and $\Lambda\left(x_{i}\right)=$ false otherwise.

Let us show that each clause $C_{j}$ has at least one true literal or one false literal. Set $C_{j}=l_{1} \vee l_{2} \vee l_{3}$. First observe that, by construction, for all $1 \leq k \leq 3, l_{k}$ is true if $\phi\left(b_{k}^{j} c^{j}\right)<$ $\phi\left(b_{k+1}^{j} c^{j}\right)$ and $l_{k}$ is false otherwise. Now the three literals are not all true otherwise, $\phi\left(b_{1}^{j} c^{j}\right)<$ $\phi\left(b_{2}^{j} c^{j}\right)<\phi\left(b_{3}^{j} c^{j}\right)<\phi\left(b_{1}^{j} c^{j}\right)$, a contradiction. And they are not all false, otherwise $\phi\left(b_{1}^{j} c^{j}\right)>$ $\phi\left(b_{2}^{j} c^{j}\right)>\phi\left(b_{3}^{j} c^{j}\right)>\phi\left(b_{1}^{j} c^{j}\right)$, a contradiction. Hence $C_{j}$ has at least one true literal and one false literal.

Reciprocally, let us now show that, if there is a truth assignment $\Lambda$ such that each clause of $I$ has at least one true literal and at least one false literal, then $G_{I}$ has a good edge-labelling.

The idea is to find a good edge-labelling $\phi$ satisfying the following property $(\star)$ : If $\Lambda\left(x_{i}\right)=$ true, $\phi\left(v_{1}^{i, j} v_{2}^{i, j}\right)<\phi\left(v_{2}^{i, j} v_{3}^{i, j}\right)$ for all $1 \leq j \leq m$ and if $\Lambda\left(x_{i}\right)=$ false, $\phi\left(v_{1}^{i, j} v_{2}^{i, j}\right)>$ $\phi\left(v_{2}^{i, j} v_{3}^{i, j}\right)$ for all $1 \leq j \leq m$.

Let $C_{j}=l_{1} \vee l_{2} \vee l_{3}$ be clause. To satisfy $(\star)$, we must label the edges of $V G_{j}$ such that $\phi\left(b_{k}^{j} c^{j}\right)<\phi\left(b_{k+1}^{j} c^{j}\right)$ if $l_{k}$ is true and $\phi\left(b_{k}^{j} c^{j}\right)>\phi\left(b_{k+1}^{j} c^{j}\right)$ if $l_{k}$ is false. As $C_{j}$ has at least one true and one false literal, there is a unique way to label the three edges $c^{j} b_{k}^{j}, 1 \leq k \leq 3$, with $\{-1,0,+1\}$ so that the condition $(\star)$ is fulfilled.

Let us now extend this edge-labelling to the remaining edges of each of the variable graphs $V G_{i}$. First, for all $1 \leq j \leq m$ and $1 \leq k \leq 4$, assign -3 and +3 alternatively on the edges of the cycle of length four containing both $r_{k}^{i, j}$ and $s_{k}^{i, j}$ so that $\phi\left(v_{k}^{i, j} r_{k}^{i, j}\right)=-3$. Then, if $\Lambda\left(x_{i}\right)=$ true, set $\phi\left(v_{3}^{i, j}, v_{4}^{i, j}\right)=-2$ and $\phi\left(v_{4}^{i, j}, v_{1}^{i, j+1}\right)=2$ for all $1 \leq j \leq m$, and, if $\Lambda\left(x_{i}\right)=$ false, set $\phi\left(v_{3}^{i, j}, v_{4}^{i, j}\right)=2$ and $\phi\left(v_{4}^{i, j}, v_{1}^{i, j+1}\right)=-2$ for all $1 \leq j \leq m$.

We claim that $\phi$ is a good edge-labelling of $G_{I}$. Suppose, by way of contradiction, that there is a pair of vertices $(x, y)$ such that two independent increasing $(x, y)$-paths $P_{1}$ and $P_{2}$ exist.

A set of two independent paths starting at a vertex of $R=\left\{r_{k}^{i, j} \mid 1 \leq j \leq m, 1 \leq k \leq\right.$ 4\} $\cup\left\{s_{k}^{i, j} \mid 1 \leq j \leq m, 1 \leq k \leq 4\right\}$ contains one increasing path (the one starting with 
the edge labelled -3 ) and one decreasing path (the one starting with the edge labelled 3). Hence $x$ and $y$ are not in $R$.

In addition, the union of $P_{1}$ and $P_{2}$ cannot be one of the four-cycles formed by the edges incident to $r_{k}^{i, j}$ and $s_{k}^{i, j}$ for some $i, j$ and $k$.

Without loss of generality, we may assume that $P_{1}$ is at least as long has $P_{2}$. As cycles formed by two graphs $G V_{i}$ and $G V_{j}$ are of length at least $6, P_{1}$ has length at least 3 . Now one can see that $P_{1}$ may not contains any vertex of $R$ because every path of length at least 3 with no end in $R$ is not increasing (nor decreasing).

Hence $P_{1}$ must contain at least three consecutive edges on one of the paths $Q_{i}=V G_{i}-R$. So $P_{1}$ is not increasing, a contradiction.

Remark 6 A standard dynamic programming approach yields a polynomial-time algorithm to decide if a graph of bounded tree-width has a good edge-labelling. It suffices to store if there is a good edge-labelling such that the ordering of edge labels corresponds to a given order (for all possible orderings of edges in the bag (there are at most $\left(\frac{k(k+1)}{2}\right)$ ! of them).

However, we do not know what is the complexity of the problem when restricted to planar graphs.

Problem 7 Does there exist a polynomial-time algorithm that decides if a given planar graph has a good edge-labelling?

\section{$5 \quad$ Classes of good graphs}

Recall that a graph $G$ is critical if it is bad but each of its proper subgraph is good. To prove that every graph in a class $\mathcal{C}$ closed under subgraphs has a good edge-labelling, it suffices to prove that $\mathcal{C}$ contains no critical graph.

Lemma 8 Every critical graph is 2-connected.

Proof: Suppose by way of contradiction that a critical graph has a cut-vertex $x$. Let $C_{1}, \ldots, C_{k}$ be the components of $G-x$ and $G_{i}=G\left[C_{i} \cup\{x\}\right], 1 \leq i \leq k$. As $G$ is critical, each of the $G_{i}$ admits a good edge-labelling. Then the union of these edge-labellings form a good edge-labelling of $G$ because there are two independent $(u, v)$-paths in $G$ only if there exists $i, 1 \leq i \leq k$, so that $u$ and $v$ are in $V\left(G_{i}\right)$. This contradicts the fact that $G$ has no good edge-labelling.

Corollary 9 Every forest $F$ admits a good edge-labelling.

Proof: No forest contains a non-trivial 2-connected subgraph, and so contains no critical subgraph. 
Let $G=(V, E)$ be a graph. An edge-2-cut of $G$ is a set of two adjacent vertices $u$ and $v$ such that $G-\{u, v\}$ has more connected components than $G$.

Lemma 10 Let $G$ be a connected graph and $\{u, v\}$ an edge-2-cut in $G$ so that $G-\{u, v\}$ has two connected components $C_{1}$ and $C_{2}$. If $G\left\langle C_{1} \cup\{u, v\}\right\rangle$ and $G\left\langle C_{2} \cup\{u, v\}\right\rangle$ have a good edge-labelling then $G$ has a good edge-labelling.

Proof: Let $\phi_{1}$ and $\phi_{2}$ be good edge-labellings of $G\left\langle C_{1} \cup\{u, v\}\right\rangle$ and $G\left\langle C_{2} \cup\{u, v\}\right\rangle$ respectively such that $\phi_{1}(u v)=\phi_{2}(u v)$.

Then the union of $\phi_{1}$ and $\phi_{2}$ is a good edge-labellign of $G$. Indeed, suppose by way of contradiction, that there exists $x$ and $y$ and two indepedent increasing $(x, y)$-paths $P_{1}$ and $P_{2}$. W. l. o. g. , we may assume that $x \in V\left(G_{1}\right)$. At least of the path, say $P_{1}$, contains at least one edge $e_{1}$ in $E\left(G_{2}\right) \backslash\{u v\}$ since $\phi_{1}$ is good.

Assume first that $y \in V\left(G_{1}\right)$. Then $P_{1}$ must go through $u$ and $v$. Let $Q_{2}$ be the shortest $(u, v)$-subpath of $P$ containing $e_{1}$. Then $Q_{2}$ is either increasing or decreasing. Hence since $u v$ is both increasing and decreasing, there are either two increasing paths or two decreasing paths in $G_{2}$. This contradicts the fact that $\phi_{2}$ is good.

Assume now that $y \in C_{2}$. Then since $P_{1}$ and $P_{2}$ are independent without loss of generality, $P_{1}$ goes through $u$ and $P_{2}$ goes through $v$. Let $Q_{1}$ be the $(x, u)$-subpath of $P_{1}, R_{1}$ be the $(u, y)$-subpath of $P_{1}$, let $Q_{2}$ be the $(x, v)$-subpath of $P_{2}$ and $R_{2}$ be the $(v, y)$-subpath of $P_{2}$.

If $\phi(u v)$ is larger than the label of the last edge of $Q_{1}$ then $R_{1} u v$ and $R_{2}$ are two increasing paths in $G_{1}$, a contradiction. If not $\phi(u v)$ is smaller than the abel of the first edge of $R_{1}$ and $v u R_{1}$ and $R_{2}$ are two increasing paths in $G_{2}$, a contradiction.

Let $G=(V, E)$ be a graph. An edge-cut is a set of edge between a set of vertices $S$ and its complement $\bar{S}$. Formally, for any $S \subset V$, the edge-cut $[S, \bar{S}]$ is the set $\{u v \in E \mid u \in$ $S$ and $v \in \bar{S})$. An edge cut which is also a matching is called a matching-cut.

Lemma 11 Let $G$ be a graph and $[S, \bar{S}]$ a matching-cut in $G$. If $G\langle S\rangle$ and $G\langle\bar{S}\rangle$ have a good edge-labelling then $G$ has a good edge-labelling.

Proof: Let $\phi_{1}$ be a good edge-labelling of $G\langle S\rangle$ and $\phi_{2}$ be a good edge-labelling of $G\langle\bar{S}\rangle$ (in $\mathbb{R})$. Then the edge-labelling $\phi$ of $G$ defined by $\phi(e)=\phi_{1}(e)$ if $e \in E(G\langle S\rangle), \phi(e)=\phi_{2}(e)$ if $e \in E(G\langle\bar{S}\rangle)$ and $\phi(e)=+\infty$ if $e \in[S, \bar{S}]$ is good.

Indeed, suppose by way of contradiction, that it is not good. Then there exist two vertices $u$ and $v$ and two independent increasing $(u, v)$-paths $P_{1}$ and $P_{2}$. Since $\phi_{1}$ and $\phi_{2}$ are good, then without loss of generality, we may assume that $u \in S$ and $v \in \bar{S}$. For $i=1,2$ $P_{i}$ contains an edge of $v_{i} w_{i}$ in $[S, \bar{S}]$. Now as $v_{1} w_{1}$ and $v_{2} w_{2}$ are labelled $+\infty$ and incident to no edges labelled $+\infty, v_{1} w_{1}$ must be the last edge of $P_{1}$ and $v_{2} w_{2}$ the last edge of $P_{2}$. So $w_{1}=v=w_{2}$ which is impossible as $[S, \bar{S}]$ is a matching. 
Corollary 12 A critical graph has no matching-cut.

Corollary 13 Every $C_{3}$-free outerplanar graph admits a good edge-labelling.

Proof: An easy result of Eaton and Hull [5] states that a $C_{3}$-free outerplanar graph has either a 1-vertex or two adjacent 2 -vertices. This implies that it has a matching-cut. Hence by Corollary 12 no $C_{3}$-free outerplanar graph is critical, which yields the result.

A graph is subcubic if every vertex has degree at most three.

Lemma 14 Every subcubic $\left\{C_{3}, K_{2,3}\right\}$-free graph has a matching cut.

Proof: Let $G$ be a subcubic graph $\left\{C_{3}, K_{2,3}\right\}$. If $G$ has no cycle, then every edge forms a matching-cut. Suppose now that $G$ has a cycle. Let $C$ be a cycle of smallest length in $G$. If $C$ is a connected component of $G$ (in particular if $C=G$ ) then any pair of non-adjacent edges of $C$ form a matching-cut.

If not, let us show that $[V(C), \overline{V(C)}]$ is a matching cut. Let $e_{1}=x_{1} y_{1}$ and $e_{2}=x_{2} y_{2}$ be two distinct edges in this set with $x_{1}, x_{2} \in V(C)$. Then $x_{1} \neq x_{2}$ because these two vertices have degree (at most) 3 and two neighbours in $V(C)$. Suppose by way of contradiction that $y_{1}=y_{2}$. Then $x_{1}$ and $x_{2}$ are not adjacent since $G$ is $C_{3}$-free. Furthermore, there the two $\left(x_{1}, x_{2}\right)$-paths along $C$ are of length at most 2 otherwise $C$ would not be a smallest cycle. Hence $C$ is a cycle of length 4 and the graph induced by $V(C) \cup\left\{y_{1}\right\}$ is a $K_{2,3}$, a contradiction.

Corollary 12 and Lemma 14 immediately implies that the sole subcubic critical graphs are $C_{3}$ and $K_{2,3}$.

Corollary 15 Every subcubic $\left\{C_{3}, K_{2,3}\right\}$-free graph has a good edge-labelling.

Farley and Proskurowski [6] proved that every (multi)graph $G$ on $n$ vertices with less than $\frac{3}{2}(n-1)$ edges has a matching-cut.

Theorem 16 (Farley and Proskurowski [6]) Let $G$ be a multigraph. If $|E(G)|<\frac{3}{2}|V(G)|-$ $\frac{3}{2}$ then $G$ has a matching-cut.

Corollary 12 and Theorem 16 yield immediately the following.

Corollary 17 Every critical graph has at least $\left\lceil\frac{3}{2}|V(G)|-\frac{3}{2}\right\rceil$ edges.

Corollary 18 Every planar graph of girth at least 6 has a good edge-labelling.

Proof: It is well known that a planar graph $G$ with girth at least 6 has at most $\frac{3}{2}|V(G)|-3$ edges. Indeed, recall the Euler's formula for a connected planar graph $G$ : $|V(G)|-|E(G)|+$ $|F(G)|=2$, with $|F(G)|$ the number of faces of $G$. Now, as $G$ has girth at least $6,6|F(G)| \leq$ $2|E(G)|$. Thus $12-6|V(G)|+6|E(G)|=6|F(G)| \leq 2|E(G)|$. Hence $|E(G)| \leq \frac{3}{2}|V(G)|-3$.

Thus no planar graph of girth 6 is critical by Corollary 17 . 


\section{Good edge-labelling of ABC-graphs}

Corollary 17 states that every critical graph has at least $\left\lceil\frac{3}{2}(|V(G)|-1)\right\rceil$ edges. This is tight since if $G$ is $C_{3}$ or $K_{2,3}$ then $|E(G)|=\left\lceil\frac{3}{2}(|V(G)|-1)\right\rceil$. We will now show that those two graphs are the unique critical ones satisfying this equality.

Farley and Proskurowski [6] constructed a class of multigraphs $G$ called $A B C$-graphs with no matching-cut having $\left\lceil\frac{3}{2}(|V(G)|-1)\right]$ edges. The definition of ABC-graphs is based on the following three operations:

- An A-operation on vertex $u$ introduces vertices $v$ and $w$ and edges $u v, u w$ and $v w$.

- A B-operation on edge $u v$ introduces vertices $w_{1}$ and $w_{2}$ and edges $u w_{1}, v w_{1}, u w_{2}$ and $v w_{2}$, and removes edge $u v$.

- A $C$-operation on vertices $u$ and $v(u=v$ is allowed) introduces vertex $w$ and edges $u w$ and $v w$.

Note that the C-operation is the only operation that can introduce parallel edges.

An $A B C$-graph is a graph that can be obtained from $K_{1}$ with a sequence of $\mathrm{A}$ - and $\mathrm{B}$-operations and at most one C-operation.

It is easy to check that ABC-graphs have no matching-cut. In addition, solving a conjecture of Farley and Proskurowski [6], Bonsma [3, 4] showed that they are the unique extremal examples, i.e., satisfying $|E(G)|=\left\lceil\frac{3}{2}(|V(G)|-1)\right\rceil$.

Theorem 19 (Bonsma $[3,4]$ ) Let $G$ be a graph with such that $|E(G)|=\left\lceil\frac{3}{2}(|V(G)|-1)\right\rceil$. Then $G$ has no matching-cut if and only if $G$ is an $A B C$-graph.

Observe that the C-operation is the only one that changes the parity of the order. Hence an ABC-graph with an odd number of vertices is obtained from $K_{1}$ with a sequence of Aand B-operations and no C-operation.

Proposition 20 Let $G$ be a graph obtained from a graph $H$ by a B-operation on some edge uv. If $H$ has a good edge-labelling then $G$ has a good edge-labelling.

Proof: Let $\phi$ be a good edge-labelling of $H$. By Proposition 2, we may assume that $\phi$ is injective. Moreover, we may assume that $\phi$ is integer-valued and $\phi(u v)=0$. Hence setting $\phi\left(u w_{1}\right)=\phi\left(w_{2} v\right)=\frac{1}{2}$ and $\phi\left(u w_{2}\right)=\phi\left(x w_{1}\right)=-\frac{1}{2}$, we obtain a good edge-labelling of $G$.

Lemma 21 Let $G$ be a 2-connected $A B C$-graph with an odd number of vertices. If $G \notin$ $\left\{C_{3}, K_{2,3}\right\}$ then $G$ has a good edge-labelling.

Proof: As every $A$-operation (with the exception of the transition $K_{1} \rightarrow C_{3}$ ) creates a cut-vertex, $G$ is obtained from $C_{3}$ with a sequence of B-operations. But a B-operation on 
$C_{3}$ at any edge creates a $K_{2,3}$ and a B-operation on $K_{2,3}$ at any edge creates the graph $G_{1}$ depicted in Figure 4. Now this graph admits a good edge-labelling (See Figure 4). Hence an easy induction and Proposition 20 implies the result because if $G \notin\left\{C_{3}, K_{2,3}\right\}$ then it is obtained from $G_{1}$ with a sequence of B-operations.

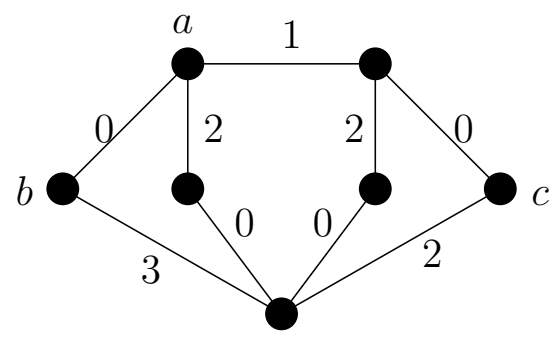

Figure 4: The graph $G_{1}$ and a good edge-labelling

Corollary 22 Let $G$ be an ABC-graph with an odd number of vertices. If $G$ is $\left\{C_{3}, K_{2,3}\right\}$ free then $G$ is good.

We now would like to prove an analogous statement to the one of Corollary 22 but for ABC-graphs with an even number of vertices.

Let $G$ be a graph and $x, y$ be two distinct vertices of $G$. An $(x, y)$-better edge-labelling of $G$ is an good edge-labelling of $G$ such that there is no increasing $(x, y)$-path. Clearly, if $x$ and $y$ are adjacent or if $x$ and $y$ have two neighbours in common then $G$ has no $(x, y)$-better edge labelling. A graph is friendly if it has a good-edge labelling and for any pair $(x, y)$ of non-adjacent vertices with at most one neighbour in common there exists an $(x, y)$-better edge-labelling.

Proposition 23 Let $G$ be a graph obtained from a graph $H$ by a B-operation on some edge $u v$. If $H$ is friendly then $G$ is friendly.

Proof: Let $w_{1}, w_{2}$ be the vertices created by the B-operation. Let $x$ and $y$ be two nonadjacent vertices of $G$ having at most one neighbour in common. Then $\left|\{x, y\} \cap\left\{w_{1}, w_{2}\right\}\right| \leq 1$.

- Suppose first that $\{x, y\} \cap\left\{w_{1}, w_{2}\right\}=\emptyset$. Then $x$ and $y$ are not adjacent in $H$.

Assume first that they have at most one commom neighbour in $H$. Let $\phi$ be an integer-valued $(x, y)$-better edge-labelling of $H$ such that $\phi(u v)=0$. Setting $\phi\left(u w_{1}\right)=$ $\phi\left(w_{2} v\right)=\frac{1}{2}$ and $\phi\left(u w_{2}\right)=\phi\left(v w_{1}\right)=-\frac{1}{2}$, we obtain an $(x, y)$-better edge-labelling of $H$.

Assume now that $x$ and $y$ have two common neighbours in $H$. As they do not have two common neighbours in $G$, we can suppose w.l.o.g. that $x=u$ and $N(x) \cap N(y)=\{v, w\}$ 
for some vertex $w$. Let $\phi$ be a good edge-labelling of $H$. By Proposition 2, we may assume that $\phi$ is integer-valued and $\phi(u v)=0$. Moreover, free to consider $-\phi$, we may assume that $\phi(v y)>0$, so $u v y$ is increasing. Hence in $H \backslash u v$ there is no increasing $(u, y)$-path. Moreover there is no increasing $(u, v)$-path (resp. increasing $(v, u)$-path) in $H \backslash u v$, otherwise there would be two such paths on $H$ because $u v$ (resp. $v u$ ) is an increasing path of $H$. Hence setting $\phi\left(u w_{1}\right)=\phi\left(w_{2} v\right)=+\infty$ and $\phi\left(u w_{2}\right)=\phi\left(x w_{1}\right)=-\infty$, we obtain an $(x, y)$-better edge-labelling of $H$.

- Suppose now that $\left|\{x, y\} \cap\left\{w_{1}, w_{2}\right\}\right|=1$. Without loss of generality, we may assume that $x=w_{1}$ and $y$ is not adjacent to $v$.

Assume first that $v$ and $y$ have at most one common neighbour in $H$. Let $\phi$ be a $(v, y)$-better edge-labelling of $H$. Then in $H \backslash u v$, there is no increasing $(u, v)$-path nor increasing $(v, u)$-path. Hence setting $\phi\left(u w_{1}\right)=\phi\left(w_{2} v\right)=+\infty$ and $\phi\left(u w_{2}\right)=$ $\phi\left(x w_{1}\right)=-\infty$, we obtain an $\left(w_{1}, y\right)$-better edge-labelling of $H$. Indeed there is no incresing $\left(w_{1}, y\right)$-path, through $u$ since $\phi\left(u w_{1}\right)=+\infty$, nor through $v$ since there is no increasing $(v, y)$-path in $H$.

Assume now that $v$ and $y$ have two common neighbours in $H$.

- Suppose that $y$ is adjacent to $u$. Let $\phi$ be a good edge-labelling of $H$. By Proposition 2, we may assume that $\phi$ is injective and integer-valued and $\phi(u v)=$ 0.

Setting $\phi\left(u w_{1}\right)=\phi\left(w_{2} v\right)=\frac{1}{2}$ and $\phi\left(u w_{2}\right)=\phi\left(x w_{1}\right)=-\frac{1}{2}$, we obtain a a $\left(w_{1}, y\right)$-better edge-labelling of $H$. In addition, free to consider $-\phi$, we may assume that $\phi(u y)<0$ and so $\phi(u y) \leq-1$. Setting $\phi\left(u w_{1}\right)=\phi\left(w_{2} v\right)=-\frac{1}{2}$ and $\phi\left(u w_{2}\right)=\phi\left(v w_{1}\right)=\frac{1}{2}$, we obtain a $\left(w_{1}, y\right)$-better edge-labelling of $H$. Indeed suppose for a contradiction that there is a $\left(w_{1}, y\right)$-increasing path $P$ :

* If $u$ is the second vertex of $P$ then $P-u$ is an increasing $(u, y)$. Since $\phi(u y) \leq-1, P$ is not $u y$. So there are two increasing $(u, y)$-paths in $H$ a contradiction.

* If $v$ is the second vertex of $P$ then the path in $H$ obtained from $P$ by replacing $w_{1}$ with $u$ is an increasing $(u, y)$-path because the labels of the edges of $P$ are positive. This path is distinct from $u y$, a contradiction.

- Suppose that $y$ is not adjacent to $u$. Let $t_{1}$ and $t_{2}$ be the two common neighbours of $v$ and $y$. Let $\phi$ be a good edge-labelling of $H$. By Proposition 2, we may assume that $\phi$ is injective and integer-valued and $\phi(u v)=0$. Moreover, without loss of generality, we may assume that $v t_{1} y$ is increasing and $v t_{2} y$ is decreasing. In addition, free to consider $-\phi$, we may assume that $\phi\left(v t_{1}\right)<0$ and so $\phi\left(v t_{1}\right) \leq$ -1 . Setting $\phi\left(u w_{1}\right)=\phi\left(w_{2} v\right)=\frac{1}{2}$ and $\phi\left(u w_{2}\right)=\phi\left(x w_{1}\right)=-\frac{1}{2}$, we obtain a $\left(w_{1}, y\right)$-better edge-labelling of $H$. Indeed suppose for a contradiction that there is a $\left(w_{1}, y\right)$-increasing path $P$ : 
* If $v$ is the second vertex of $P$ then $P-w_{1}$ is an increasing $(v, y)$. Since $\phi\left(v t_{1}\right) \leq-1, P$ is not $v t_{1} y$. So there are two increasing $(v, t)$-path in $H$ a contradiction.

* If $u$ is the second vertex of $P$ then the path in $H$ obtained from $P$ by replacing $w_{1}$ with $v$ is an increasing $(v, y)$-path because the labels of the edges of $P$ are positive. This path is distinct from $v t_{1} y$, a contradiction.

One can now generalise Lemma 21.

Lemma 24 Let $G$ be a 2-connected $A B C$-graph with an odd number of vertices. If $G \notin$ $\left\{C_{3}, K_{2,3}\right\}$ then $G$ is friendly.

Proof: Similarly as in the proof of Lemma 21, it suffices to prove that $G_{1}$ is friendly. Then an easy induction and Proposition 23 yield the result.

Let $x$ and $y$ be two vertices of $G_{1}$ that are not adjacent and that have at most one neighbour in common, and let $\phi$ be the labelling of $G_{1}$ given in Figure $4: \phi$ or $-\phi$ is a $(x, y)$-better edge labelling.

Corollary 25 Every $\left\{C_{3}, K_{2,3}\right\}$-free $A B C$-graph with an odd number of vertices is friendly.

Proof: Let $x$ and $y$ be two non-adjacent vertices of $G$ having at most one common neighbour.

Assume first that $x$ and $y$ are in a same connected 2-component $C$. By Lemma 24, $C$ has an $(x, y)$-better labelling and, by Corollary $22, G \backslash E(C)$ has a good edge-labelling. The union of these two edge-labellings is clearly an $(x, y)$-better labelling of $G$.

Suppose now that the 2-connected components containing $x$ do not contain $y$. Let $G_{1}$ be the graph induced by the union of the 2-connected components containing $x$ and $G_{2}=G \backslash E\left(G_{1}\right)$. By Corollary 22, the two graphs $G_{1}$ and $G_{2}$ admit good edge-labellings $\phi_{1}$ and $\phi_{2}$, respectively. Free to add a huge number to all the labels of $\phi_{1}$, we may assume that $\min \left\{\phi_{1}(e) \mid e \in E\left(G_{1}\right)\right\}>\max \left\{\phi_{2}(e) \mid e \in E\left(G_{2}\right)\right\}$. Then the union of $\phi_{1}$ and $\phi_{2}$ is an $(x, y)$-better labelling of $G$.

Lemma 26 Let $G$ be an $A B C$-graph with an even number of vertices. If $G$ is $\left\{C_{3}, K_{2,3}\right\}$-free then $G$ is good.

Proof: We prove this lemma by induction on the number of vertices (or equivalently the number of A-, B- or C-operations). An even ABC-graph is obtained from $K_{1}$ with a sequence of A- and B-operations and exactly one C-operation. If $G$ is not 2-connected then we get the result as in the proof of Lemma 8. So we may assume that $G$ is 2 -connected. In this case there can be no A-operation made after a $\mathrm{C}$-operation. Consider a sequence of operations such that the $\mathrm{C}$-operation is done as late as possible. 
If the $\mathrm{C}$-operation is the ultimate one, say it is done on $u$ and $v$, and introduces $w$. Note that $u \neq v$ since $G$ has no multiple edges. Since $G$ is $\left\{C_{3}, K_{2,3}\right\}$-free then $u$ and $v$ are not adjacent and $u$ and $v$ have at most one neighbour in common. Hence by Corollary 25, $G-w$ admits a $(u, v)$-better edge-labelling $\phi$ (in $\mathbb{R})$. Setting $\phi(u w)=-\infty$ and $\phi(w v)=+\infty$ we obtain a good edge-labelling of $G$.

If the C-operation is the penultimate one, then it is followed by a B-operation on one of the introduced edges. These two operations together may be seen as a single one on $u$ and $v$ that introduces the vertices $t_{1}, t_{2}$ and $w$ and the edges $u t_{1}, u t_{2}, t_{1} w, t_{2} w$ and $w v$.

Note that $u$ and $v$ are not adjacent since $G$ is $K_{2,3}$-free. Assume first that $u$ and $v$ have at most one neighbour in common. By Corollary $25, G-\left\{t_{1}, t_{2}, w\right\}$ admits a $(u, v)$-better edge-labelling $\phi$. Let $M$ be the maximum value of $\phi$. Then setting $\phi\left(u t_{1}\right)=\phi\left(t_{2} w\right)=-\infty$, $\phi\left(u t_{2}\right)=\phi\left(t_{1} w\right)=M+1$ and $\phi(v w)=M+2$, we obtain a good edge-labelling of $G$.

Assume now that $u$ and $v$ have at least two common neighbours. Since $G$ is $K_{2,3^{-}}$ free, then $u$ and $v$ have exactly two common neighbours $x_{1}$ and $x_{2}$. By Corollary 22, $G-\left\{t_{1}, t_{2}, w\right\}$ admits a good edge-labelling $\phi$. By Proposition 2, we may assume that $\phi$ is injective. Without loss of generality, we may suppose that $\phi\left(v x_{1}\right)>\phi\left(v x_{2}\right)$. Let us set $\phi\left(u t_{1}\right)=\phi\left(t_{2} w\right)=+\infty, \phi\left(u t_{2}\right)=\phi\left(t_{1} w w\right)=-\infty$ and $\phi(v w)=\frac{1}{2}\left(\phi\left(v x_{1}\right)+\phi\left(v x_{2}\right)\right)$. We claim that $\phi$ is a good edge-labelling of $G$. Indeed suppose, by way of contradiction, that it is not the case. Then there exist two vertices $a$ and $b$ and two independent increasing $(a, b)$-paths $P_{1}$ and $P_{2}$. Since $\phi$ is a good edge-labelling of $G-\left\{t_{1}, t_{2}, w\right\}$ one of these two paths, say $P_{1}$ must go through $w$. Moreover since $\phi\left(t_{1} w\right)=-\infty$ and $\phi\left(t_{2} w\right)=+\infty w$, and $d(w)=3$, then either $w t_{1}$ is the first edge of $P_{1}$ or $t_{2} w$ is the last edge of $P_{1}$. Free to consider $-\phi$ instead of $\phi$, we may assume that we are in the first case. So $P_{1}=\left(w, t_{1}, u\right)$, because $\phi\left(u t_{1}\right)=+\infty$. Now the first edge of $P_{2}$ is $w v$. Hence $Q_{2}=P_{2}-w$ is an increasing $(v, u)$-path and $v x_{2}$ is not the first edge of $Q_{2}$ since $\phi(w v)>\phi\left(v x_{2}\right)$. Note that by Observation 3.1, $v x_{2} u$ is increasing because $\phi\left(v x_{1}\right)>\phi\left(v x_{2}\right)$. So, in $G-\left\{t_{1}, t_{2}, w\right\}$, there are two distinct increasing $(v, u)$-paths. This contradicts the fact that $\phi$ is a good edge-labelling of $G-\left\{t_{1}, t_{2}, w\right\}$.

If there are exactly two B-operations after the C-operation, and if $u$ and $v$ are not adjacent then by the induction hypothesis and Proposition 20, $G$ has a good edge-labelling. If $u$ and $v$ are adjacent, then $u v$ is an edge-2-cut. Let $C_{1}$ be the component of $G-\{u, v\}$ containing $w$ (i.e., the set of vertices added with the C-operations and the following B-operations). Let $G_{1}=G\left\langle C_{1} \cup\{u, v\}\right\rangle$ and $G_{2}=G\left\langle V(G) \backslash C_{1}\right\rangle$. Note that $G_{1}$ is obtained from a triangle by performing two B-operations and thus is the graph $G_{1}$ depicted Figure 4 which has a good edge-labelling. Similarly, $G_{2}$ is the graph $G$ taken before peforming the C-operation, is so $\left\{C_{3}, K_{2,3}\right\}$-free and by Corollary 22 has a good edge-labelling. Hence by Lemma 10, $G$ has a good edge-labelling.

If there are at least three $\mathrm{B}$-operations after the $\mathrm{C}$-operation, then by the induction hypothesis and Lemma 20, $G$ has a good edge-labelling.

Corollary 22 and Lemma 26 immediately yield the following.

Theorem 27 Let $G$ be a critical graph. If $G \notin\left\{C_{3}, K_{2,3}\right\}$ then $|E(G)| \geq \frac{3}{2}\left(|V(G)|-\frac{1}{2}\right)$. 


\section{Conclusions and further research}

The average degree of a graph $G$ is $A d(G)=\frac{\sum_{v \in V(G)} d(v)}{|V(G)|}=\frac{2|E(G)|}{|V(G)|}$

Theorem 27 implies that for any $c<3$ there is a finite number of critical graphs with average degree at most $c$. Actually, we conjecture that the only ones are $C_{3}$ and $K_{2,3}$.

Conjecture 28 Let $G$ be a critical graph. Then $A d(G) \geq 3$ unless $G \in\left\{C_{3}, K_{2,3}\right\}$.

More generally for any $c<4$, we conjecture the following.

Conjecture 29 For any $c<4$, there exists a finite list of graphs $\mathcal{L}$ such that if $G$ is a critical graph with $A d(G) \leq c$ then $G \in \mathcal{L}$.

The constant 4 in the above conjecture would be tight. Indeed, for all $k$, the graph $J_{2,2, k}$ defined in Section 3 is critical: it is bad according to Proposition 4. Moreover one can easily show that for any edge $e, H_{k} \backslash e$ has a good edge-labelling with no $(u, v)$-increasing path and no $(v, u)$-increasing (just follow the constraint as in the proof of Proposition 3). Extending this labelling by labelling the two $H_{2}$ with $-\infty$ and $+\infty$ so that one of them is an increasing $(u, v)$-path and the other one an increasing $(v, u)$-path we obtain a good edge-labelling of $J_{2,2, k} \backslash e$. Furthermore $A d\left(J_{2,2, k}\right)=\frac{8 k+8}{2 k+4}=4-\frac{4}{k+2}$. Last, one can easily see that if $k \neq k^{\prime}$ then $J_{2,2, k}$ is not a subgraph of $J_{2,2, k^{\prime}}$.

Theorem 27 says that if a graph has no dense subgraphs then it has a good edge-labelling. On the opposite direction one may wonder what is the minimum density ensuring a graph to be bad. Or equivalently,

Problem 30 What is the maximum number $g(n)$ of edges of a good graph on $n$ vertices?

Clearly we have $g(n)=e x(n, \mathcal{C})$ where $\mathcal{C}$ is the set of critical graphs. As $K_{2,3}$ is critical then $g(n) \leq e x\left(n, K_{2,3}\right)=\frac{1}{\sqrt{2}} n^{3 / 2}+O\left(n^{4 / 3}\right)$ by a result of Füredi [7].

The hypercubes show that $g$ is super-linear. Indeed the hypercube $H_{k}$ is obtained from two disjoints copies of $H_{k-1}$ by adding a perfect matching between them. Hence an easy induction and Lemma 11 shows that $H_{k}$ has a good edge-labelling. Since $H_{k}$ has $2^{k}$ vertices and $2^{k-1} k$ edges, $g\left(2^{k}\right) \geq 2^{k-1} k$, so $g(n) \geq \frac{1}{2} n \log n$.

\section{References}

[1] Bala, K., And Stern, T. Algorithms for routing in a linear lightwave network. In IEEE INFOCOM'91. Proceedings. Tenth Annual Joint Conference of the IEEE Computer and Communications Societies. Networking in the 90s. (1991), pp. 1-9.

[2] Bermond, J.-C., Cosnard, M., And Pérennes, S. Directed acyclic graphs with unique path property. Tech. Rep. RR-6932, INRIA, May 2009. 
[3] Bonsma, P. A characterization of extremal graphs with no matching-cut. In Proceedings of the European conference on combinatorics, graph theory and applications (EuroComb 05) (Nancy, 2005), Discrete Mathematics and Theoretical Computer Science Proceedings AE, pp. 135-138.

[4] Bonsma, P. A characterization of extremal graphs with no matching-cut. Tech. Rep. Memorandum 1769, University of Twente, 2005.

[5] Eaton, N., And Hull, T. Defective list colorings of planar graphs. Bull. Inst. Combin. Appl 25 (1999), 79-87.

[6] FArley, A., And Proskurowski, A. Extremal graphs with no disconnecting matching. In Proceedings of the second West Coast conference on combinatorics, graph theory, and computing (1984), pp. 153-165.

[7] Füredi, Z. New asymptotics for bipartite Turán numbers. J. Combin. Theory Ser. A 75, 1 (1996), 141-144.

[8] MukherJee, B. Optical communication networks. McGraw-Hill New York, 1997.

[9] Ramaswami, R., and Sivarajan, K. Routing and wavelength assignment in alloptical networks. IEEE/ACM Transactions on Networking (TON) 3, 5 (1995), 489500.

[10] Schaefer, T. J. The complexity of satisfiability problems. In STOC 'r8: Proceedings of the tenth annual ACM symposium on Theory of computing (New York, NY, USA, 1978), ACM, pp. 216-226.

[11] SEREnI, J.-S. Private communication. 
Unité de recherche INRIA Sophia Antipolis 2004, route des Lucioles - BP 93 - 06902 Sophia Antipolis Cedex (France)

Unité de recherche INRIA Futurs : Parc Club Orsay Université - ZAC des Vignes 4, rue Jacques Monod - 91893 ORSAY Cedex (France)

Unité de recherche INRIA Lorraine : LORIA, Technopôle de Nancy-Brabois - Campus scientifique 615, rue du Jardin Botanique - BP 101 - 54602 Villers-lès-Nancy Cedex (France)

Unité de recherche INRIA Rennes : IRISA, Campus universitaire de Beaulieu - 35042 Rennes Cedex (France)

Unité de recherche INRIA Rhône-Alpes : 655, avenue de l'Europe - 38334 Montbonnot Saint-Ismier (France) Unité de recherche INRIA Rocquencourt : Domaine de Voluceau - Rocquencourt - BP 105 - 78153 Le Chesnay Cedex (France) 